\title{
Comparison Between FS-MPC Control Strategy for an UPS inverter application in $\alpha-\beta$ and abc frames
}

\author{
S. Vazquez, \\ J.I. Leon, L.G. Franquelo, \\ J.M. Carrasco and E. Dominguez, \\ Escuela Superior de Ingenieros. Electronics Engineering Department \\ University of Seville \\ Avd. Camino de los descubrimientos s/n CP: 41092 \\ Seville, Spain \\ Email: svazquez@gte.esi.us.es
}

\begin{abstract}
The voltage source inverter (VSI) of an uninterruptible power supply (UPS) is a system where the main objective is to obtain a high quality output sinusoidal voltage with independence on the output load. For this reason, it includes an output LC filter. The presence of the filter increases the complexity of the controller design thus it is necessary to evaluate the performance of the control strategy in terms of the output voltage quality and computational cost of the algorithm. In this paper, both analysis are developed for the finite states model predictive control (FSMPC) of a VSI performed in the abc and $\alpha-\beta$ frames. Both algorithms are summarized and compared in order to establish an objective criteria to choose among them when a hardware implementation is developed. Simulation results are presented for both algorithms to validate the analysis.
\end{abstract}

\section{INTRODUCTION}

The voltage source inverter (VSI) of an uninterruptible power supply (UPS) is a system where the main objective is to obtain a high quality output sinusoidal voltage with independence on the output load. For this reason, the VSI includes an output LC filter to remove the high order harmonic components of the output voltage due to the switching of the power semiconductors. The inclusion of this filter increases the complexity of the system controller design, resulting in control strategies with high computational cost [1]-[11].

Predictive control is a sort of control strategy that can be applied for the control of power converters [12]-[13]. Within predictive control, model predictive control (MPC) is based on the use of a model of the system to predict the behavior of the converter variables until certain horizon of time. Then, the optimal control action is selected by minimizing a cost function, [14]. MPC is a very flexible control scheme that allows easy inclusion of system constraints and nonlinearities in the design stage of the controller. Besides, MPC can be developed considering the inputs of the system model as continuous variables [15] or as a set of finite states (FS) [16].

FS-MPC approach has been used to develop the control of a VSI for an UPS application in the $\alpha-\beta$ frame [16]-[17]. In this work, a comparison between the FS-MPC control strategy for an UPS inverter application in the $\alpha-\beta$ and the abc frames is developed. Both algorithms are assessed from the point of

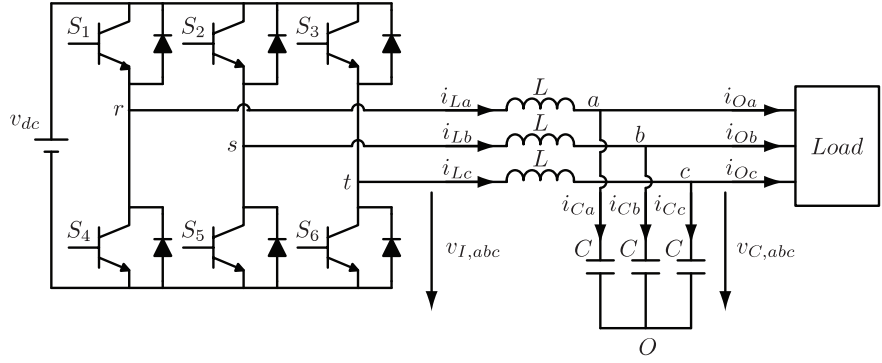

Fig. 1. Three-phase inverter with $L C$ filter.

TABLE I

SYSTEM VARIABLES AND PARAMETERS

\begin{tabular}{|c|c|}
\hline Variable & Description \\
\hline$v_{C, a b c}=\left\{v_{a O} v_{b O} v_{c O}\right\}^{T}$ & Output filter capacitor voltage vector \\
\hline$i_{L, a b c}=\left\{i_{L a} i_{L b} i_{L c}\right\}^{T}$ & Output inductor current vector \\
\hline$v_{I, a b c}=\left\{v_{r O} v_{s O} v_{t O}\right\}^{T}$ & VSI output voltage vector \\
\hline$i_{O, a b c}=\left\{i_{O a} i_{O b} i_{O c}\right\}^{T}$ & Output load current vector \\
\hline$S_{a b c}=\left\{S_{a} S_{b} S_{c}\right\}^{T}$ & Switching vector \\
\hline$\left.S_{p}\right|_{p=\{a, b, c\}}=\{-1,1\}$ & Switching functions \\
\hline$L$ & Output filter inductance \\
\hline$C$ & Output filter capacitance \\
\hline$v_{d c}$ & dc-link voltage \\
\hline
\end{tabular}

view of the quality of the output voltage, which is evaluated through the total harmonic distortion (THD) of the output voltage. Besides, the computational cost of both algorithms are calculated in order to evaluate which method provides best performance with the lower number of calculations.

\section{SYSTEM DESCRIPTION}

A three-phase two-level power converter used as a VSI is depicted in Fig. 1. The VSI output voltages have high order harmonic components due to the switching of the power semiconductors. Thus the converter is connected to the load through a $L C$ filter in order to provide a high quality sinusoidal voltage. The system parameters and variables are described in Table I. 


$$
\begin{gathered}
i_{L, a b c}=C \frac{d v_{C, a b c}}{d t}+i_{O, a b c} \\
v_{I, a b c}=L \frac{d i_{L, a b c}}{d t}+v_{C, a b c} \\
v_{I, a b c} \triangleq \frac{v_{d c}}{2} P S_{a b c} \\
P=\frac{1}{3}\left[\begin{array}{rrr}
2 & -1 & -1 \\
-1 & 2 & -1 \\
-1 & -1 & 2
\end{array}\right]
\end{gathered}
$$

The behavior of the system can be described in the abc frame through the dynamic equations of the output filter inductor currents (1) and the output filter capacitor voltages (2). These dynamics are functions of the VSI output voltages that depend on the power semiconductors switching functions and can be calculated by (3)

$$
\begin{gathered}
m_{\alpha \beta}=T_{a b c}^{\alpha \beta} m_{a b c} \\
T_{a b c}^{\alpha \beta}=\sqrt{\frac{2}{3}}\left[\begin{array}{ccc}
1 & -\frac{1}{2} & -\frac{1}{2} \\
0 & \frac{\sqrt{3}}{2} & -\frac{\sqrt{3}}{2}
\end{array}\right]
\end{gathered}
$$

Besides, the inductor currents and the capacitor voltages can be expressed in the $\alpha \beta$ frame by means of the clarke's transformation (5)-(6) yielding

$$
\begin{aligned}
& i_{L, \alpha \beta}=C \frac{d v_{C, \alpha \beta}}{d t}+i_{O, \alpha \beta} \\
& v_{I, \alpha \beta}=L \frac{d i_{L, \alpha \beta}}{d t}+v_{C, \alpha \beta} .
\end{aligned}
$$

\section{FS-MPC UPS INVERTER CONTROL STRATEGY}

The FS-MPC control strategy of the VSI for the UPS application is based on predicting the behavior of the system for each switching vector and to choose the control action as the switching vector that minimize a certain cost function. This control function is built in such a way that includes all the control objectives and constrains that are necessary to achieve the desired behavior of the system [14].

The set of switching vectors evaluated is constituted by all the possible combinations of switching functions for the power semiconductors. In this paper the number of combinations is eight, as a conventional three-phase two-level converter topology is considered for the inverter. Two combinations produce the same output voltage vector, corresponding to the simultaneous connection of the three upper switches or the simultaneous connection of the three lower switches. Although one of these switching vectors can be excluded when a simple cost function is considered, which is the case in this work, both vectors have been maintained in the analysis to provide generality to the conclusions.

\section{A. Control algorithm in abc frame}

The control algorithm in the abc frame can be obtained from the output filter inductor currents and capacitor voltages dynamics (1)-(2). To develop the algorithm these equations are expressed in the state space, taking as state variables the output filter inductor currents and capacitor voltages.

$$
\begin{gathered}
A^{a b c}=\left[\begin{array}{cc}
0_{3 x 3} & -\frac{1}{L} I_{3 x 3} \\
\frac{1}{C} I_{3 x 3} & 0_{3 x 3}
\end{array}\right] \\
B^{a b c}=\left[\begin{array}{cc}
\frac{1}{L} I_{3 x 3} & 0_{3 x 3} \\
0_{3 x 3} & 0_{3 x 3}
\end{array}\right] ; B_{d}^{a b c}=\left[\begin{array}{rr}
0_{3 x 3} & 0_{3 x 3} \\
0_{3 x 3} & -\frac{1}{C} I_{3 x 3}
\end{array}\right] \\
I_{3 x 3}=\left[\begin{array}{lll}
1 & 0 & 0 \\
0 & 1 & 0 \\
0 & 0 & 1
\end{array}\right] ; 0_{3 x 3}=\left[\begin{array}{ccc}
0 & 0 & 0 \\
0 & 0 & 0 \\
0 & 0 & 0
\end{array}\right] \\
B_{q}^{a b c}=\int_{0}^{a b c} e^{A^{a b c} \tau} B^{a b c} d \tau ; B^{A^{a b c} T_{s}} \\
\end{gathered}
$$

Taking into account the definitions of the matrices $A^{a b c}$, $B^{a b c}$ and $B_{d}^{a b c}$ presented in (9)-(10), the state space continuous model can be transformed to its state space discrete model using (12)-(13), [18]. Thus, the final expression used to predict the state variables is

$$
x_{a b c}(k+1)=A_{q}^{a b c} x_{a b c}(k)+B_{q}^{a b c} \bar{v}_{I, a b c}(k)+B_{d q}^{a b c} \bar{i}_{O, a b c}(k),
$$

where the following vectors are used

$$
\begin{aligned}
x_{a b c} & =\left\{\begin{array}{lll}
i_{L, a b c} & v_{C, a b c}
\end{array}\right\}^{T} \\
\bar{v}_{I, a b c} & =\left\{\begin{array}{llll}
v_{I, a b c} & 0 & 0 & 0
\end{array}\right\}^{T} \\
\bar{i}_{O, a b c} & =\left\{\begin{array}{llll}
0 & 0 & 0 & i_{O, a b c}
\end{array}\right\}^{T} .
\end{aligned}
$$

As can be observed, the output load currents vector is needed to predict the system behavior. These currents can be measured or can be estimated by using an observer [17]. For simplicity in this work the first option is chosen. Besides, it is assumed that the load currents change slowly compared to the sampling frequency [17]. As a consequence, if the prediction horizon is small enough then these currents can be approximated as constants over the prediction horizon. In this work, only one time step horizon is considered thus it is possible to make the following assumption

$$
i_{O, a b c}(k+1)=i_{O, a b c}(k) .
$$

Finally, a cost function should be defined to perform the evaluation of the possible switching vectors. In this case a very simple cost function has been considered. This function appraises the sum of all square errors between the reference and the predicted output capacitor voltage vectors.

$$
g_{a b c}(k) \triangleq \sum_{p=\{a, b, c\}}\left(v_{C, p}^{*}(k+2)-v_{C, p}(k+2)\right)^{2} .
$$

The complete control algorithm in the abc frame is shown in Fig. 2, where the references to the equations used to calculate the different variable values are also displayed. 


\section{B. Control algorithm in $\alpha-\beta$ frame}

The control algorithm in the $\alpha-\beta$ frame is developed from the output filter dynamics in the stationary reference frame (7)-(8). This algorithm is obtained following the same process as for the abc frame case. Thus, the first step is to express the output filter inductor currents and capacitor voltages dynamics in the state space, taking as state variables the output filter inductor currents and capacitor voltages.

$$
\begin{gathered}
A^{\alpha \beta}=\left[\begin{array}{cr}
0_{2 x 2} & -\frac{1}{L} I_{2 x 2} \\
\frac{1}{C} I_{2 x 2} & 0_{2 x 2}
\end{array}\right] \\
B^{\alpha \beta}=\left[\begin{array}{cc}
\frac{1}{L} I_{2 x 2} & 0_{2 x 2} \\
0_{2 x 2} & 0_{2 x 2}
\end{array}\right] ; B_{d}^{\alpha \beta}=\left[\begin{array}{rr}
0_{2 x 2} & 0_{2 x 2} \\
0_{2 x 2} & -\frac{1}{C} I_{2 x 2}
\end{array}\right] \\
I_{2 x 2}=\left[\begin{array}{cc}
1 & 0 \\
0 & 1
\end{array}\right] ; 0_{2 x 2}=\left[\begin{array}{cc}
0 & 0 \\
0 & 0
\end{array}\right] \\
A_{q}^{\alpha \beta}=e^{A^{\alpha \beta} T_{s}} \\
B_{q}^{\alpha \beta}=\int_{0}^{T s} e^{A^{\alpha \beta} \tau} B^{\alpha \beta} d \tau ; B_{d q}^{\alpha \beta}=\int_{0}^{T s} e^{A^{\alpha \beta} \tau} B_{d}^{\alpha \beta} d \tau .
\end{gathered}
$$

Then, taking into account the definitions of the matrices $A^{\alpha \beta}, B^{\alpha \beta}$ and $B_{d}^{\alpha \beta}$, (20)-(21), the state space continuous model should be transformed to its state space discrete model using (23)-(24). Yielding the final expression used to predict the state variables

$$
x_{\alpha \beta}(k+1)=A_{q}^{\alpha \beta} x_{\alpha \beta}(k)+B_{q}^{\alpha \beta} \bar{v}_{I, \alpha \beta}(k)+B_{d q}^{\alpha \beta} \bar{i}_{O, \alpha \beta}(k),
$$

where the following vectors are used

$$
\begin{aligned}
x_{\alpha \beta} & =\left\{\begin{array}{ll}
i_{L, \alpha \beta} & v_{C, \alpha \beta}
\end{array}\right\}^{T} \\
\bar{v}_{I, \alpha \beta} & =\left\{\begin{array}{lll}
v_{I, \alpha \beta} & 0 & 0
\end{array}\right\}^{T} \\
\bar{i}_{O, \alpha \beta} & =\left\{\begin{array}{lll}
0 & 0 & i_{O, \alpha \beta}
\end{array}\right\}^{T} .
\end{aligned}
$$

Again the output load currents vector is needed to predict the system behavior. To develop a comparison under the same conditions, the same assumptions as for control algorithm in abc frame are adopted. That is, the output load currents are measured and are considered as constants over the prediction horizon thus

$$
i_{O, \alpha \beta}(k+1)=i_{O, \alpha \beta}(k) .
$$

Finally, the cost function for the control algorithm in the $\alpha-\beta$ frame is defined as simple as for the abc frame. This function assesses the sum of all square errors between the reference and the predicted output capacitor voltage vectors

$$
g_{\alpha \beta}(k) \triangleq \sum_{p=\{\alpha, \beta\}}\left(v_{C, p}^{*}(k+2)-v_{C, p}(k+2)\right)^{2} .
$$

The complete control algorithm in the $\alpha-\beta$ frame is shown in Fig. 3, where the references to the equations used to calculate the different variable values are included.

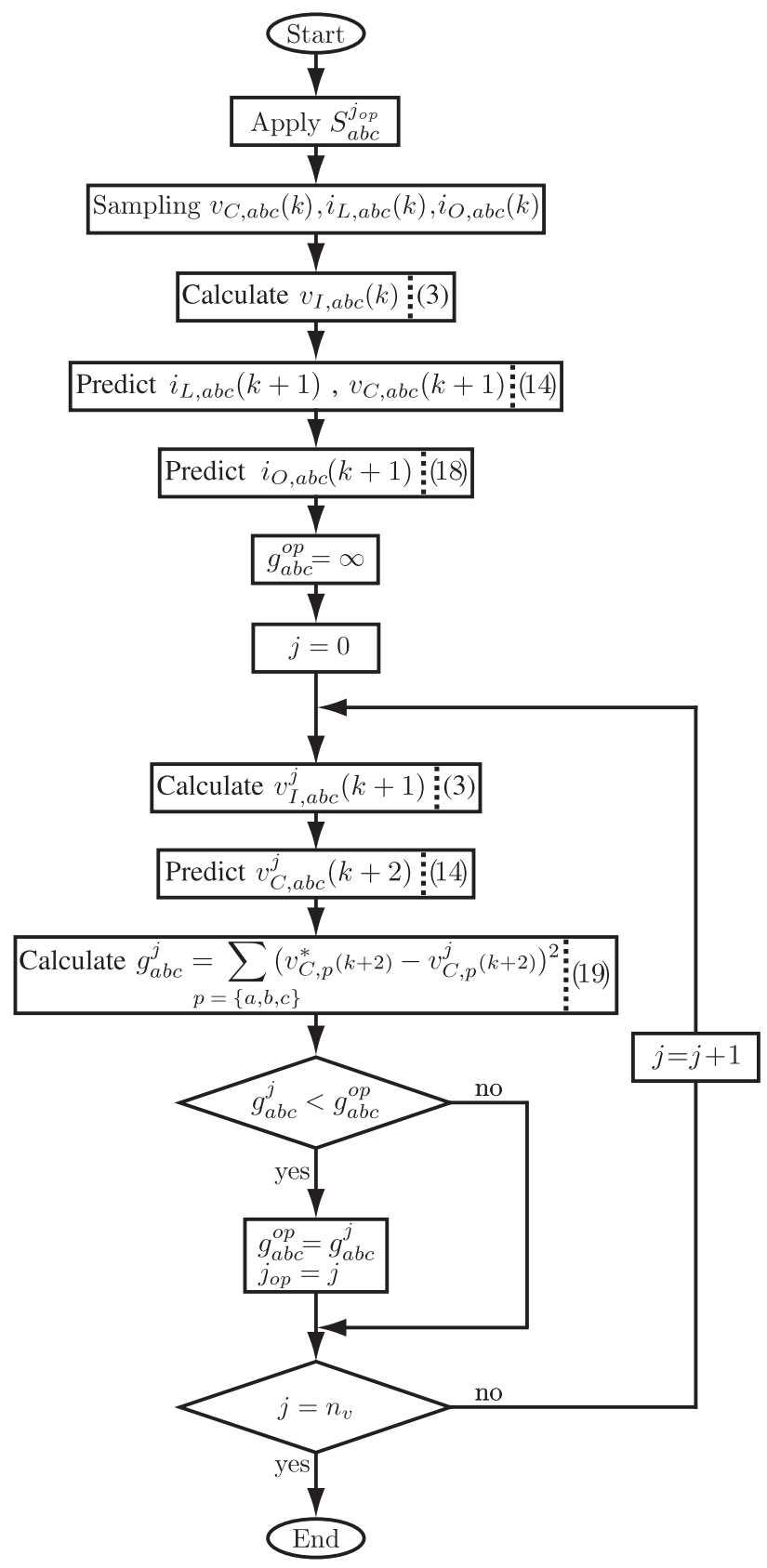

Fig. 2. FS-MPC algorithm in the abc frame.

\section{Comparison: abc frame vs $\alpha-\beta$ frame}

Two different points of view have been adopted to compare both algorithms. First of all, the quality of the output voltages obtained with both algorithms are evaluated. This quality is measured using the THD of the output voltage as indicator. On the other hand, the number of operations needed to perform the algorithms is quantified. Then the computational costs are compared in terms of which algorithm needs more effort to achieve the desired output voltage.

To develop these studies, several simulations using PSCAD/EMTDC software tool have been done. The system parameters used in the simulation are summarized in Table II. 


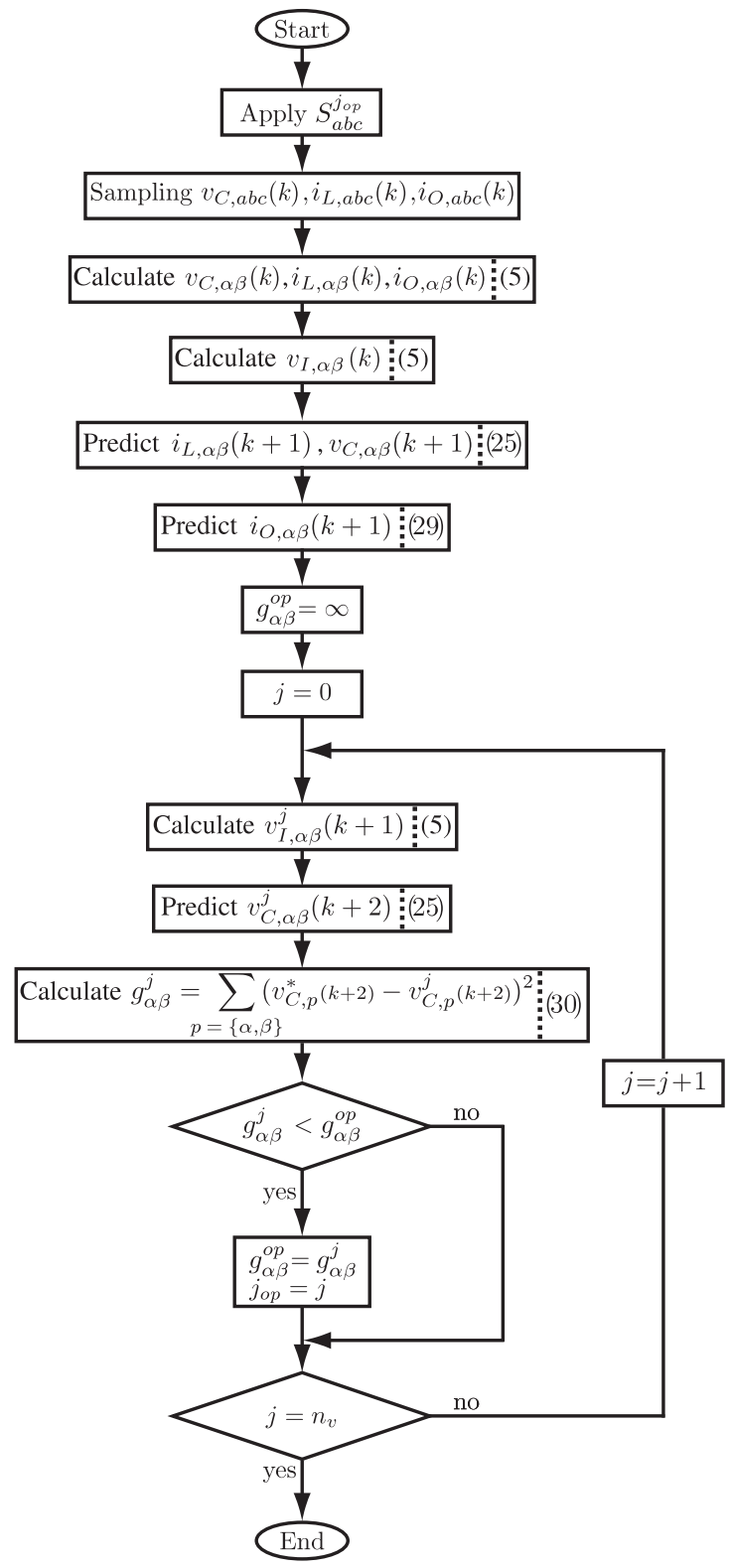

Fig. 3. FS-MPC algorithm in the $\alpha-\beta$ frame.

TABLE II

SimUlation PARAMETERS.

\begin{tabular}{cc}
\hline Parameter & Value \\
\hline dc-link voltage $\left(v_{d c}\right)$ & $520 \mathrm{~V}$ \\
Output filter inductance $(L)$ & $2.4 \mathrm{mH}$ \\
Output filter capacitance $(C)$ & $40 \mu \mathrm{F}$ \\
Sampling frequency $\left(f_{s}\right)$ & $10 \mathrm{kHz}$ \\
Output voltage references $\left(v_{C, a b c}^{*}\right)$ & $150 \mathrm{VRMS}$ \\
\hline \hline
\end{tabular}

TABLE III

THD OF THE OUTPUT VOLTAGE.

\begin{tabular}{ccc}
\hline \hline & abc frame & $\alpha \beta$ frame \\
\hline Linear load & $1.54 \%$ & $1.54 \%$ \\
Non-inear load & $2.56 \%$ & $2.56 \%$ \\
\hline \hline
\end{tabular}
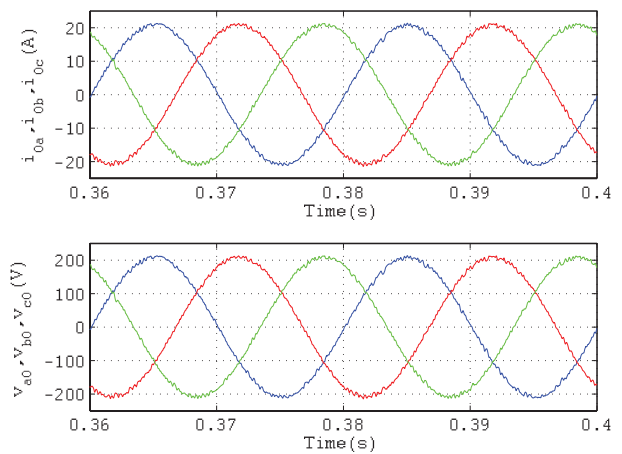

Fig. 4. Control in abc frame under linear load.
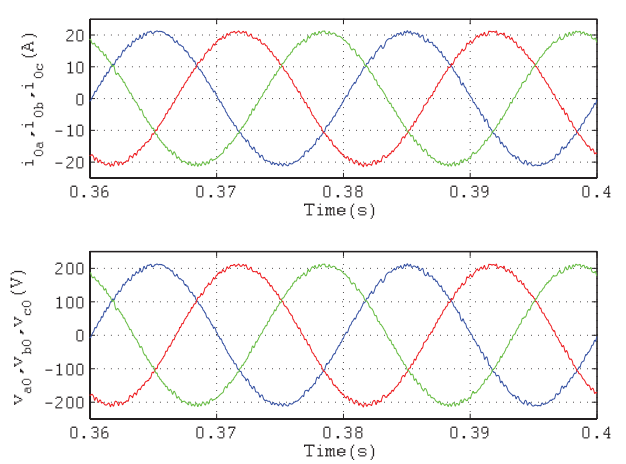

Fig. 5. Control in $\alpha \beta$ frame under linear load.
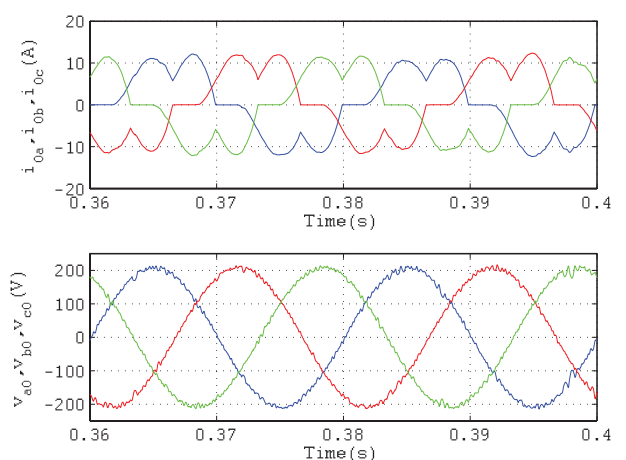

Fig. 6. Control in abc frame under non-linear load.
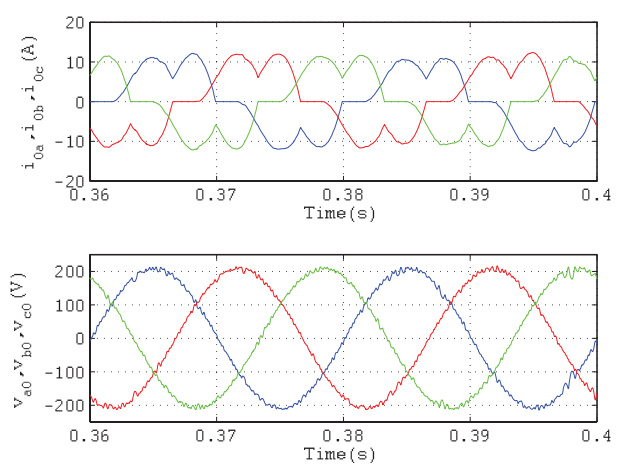

Fig. 7. Control in $\alpha \beta$ frame under non-linear load. 
TABLE IV

NUMBER OF OPERATIONS

\begin{tabular}{ccc}
\hline \hline Operation & abc frame & $\alpha \beta$ frame \\
\hline Sum & 76 & 51 \\
Subtraction & 78 & 58 \\
Product & 198 & 141 \\
\hline
\end{tabular}

Two different simulations have been considered for each control algorithm, VSI operation under linear load and VSI operation under non-linear load. As linear load, a resistor of $10 \Omega$ has been adopted for each phase. The resistors have been connected using a wye configuration. For the non-linear load operation, a single-phase diode bridge has been used for each phase. These non-controlled rectifiers are characterized by a smoothing inductor of $10 \mathrm{mH}$, a dc-link capacitor of $470 \mu \mathrm{F}$ and a $100 \Omega$ resistor as dc load. Besides, the diode bridges are connected using a delta configuration.

The voltage and current waveforms obtained for the control algorithm in the abc frame are shown in Fig. 4 and Fig. 6. Whereas the results for the control algorithm in the $\alpha$ - $\beta$ frame are shown in Fig. 5 and Fig. 7. The output voltage THD reached in each of these simulations are presented in Table III. It can be observed that the same THDs are achieved under the different VSI operation conditions with independence on the algorithm used. This result is as expected because the coordinate transformation to other stationary frame should not diminish the performance of the FS-MPC controller. Thus the computational efforts will point out which algorithm is preferred.

To assess the computational costs of the algorithms, the total number of operations needed to develop each one are counted. The sort of operations considered are sums, subtractions and products. However, the assignments have been neglected. The total number of operations can be calculated from the equations involved in the control algorithms shown in Fig. 2 and Fig. 3 for the abc and $\alpha-\beta$ frames respectively.

The number of these type of operations are summarized in Table IV and plotted in Fig. 8 for ease of comparison. The operations are presented individually because the number of clock cycles required to develop each operation depends on the selected control hardware, used to perform the implementation of the system. For example, in an TMS320C28341 each of these operations needs two pipeline cycles [19]. This supposes a total number of 704 cycles for the abc frame algorithm and 500 cycles for the $\alpha-\beta$ frame algorithm. As a consequence, if the control strategy is performed in the $\alpha-\beta$ frame a roughly computational cost reduction of $30 \%$ can be achieved. Thus, it is possible to choose a cheaper hardware platform due to the lower requirements of the control algorithm.

\section{CONCLUSIONS}

The main objective of the voltage source inverter (VSI) of an uninterruptible power supply (UPS) is to achieve a high quality sinusoidal output voltage under linear and non-linear loads, including for this purpose an output LC filter to remove

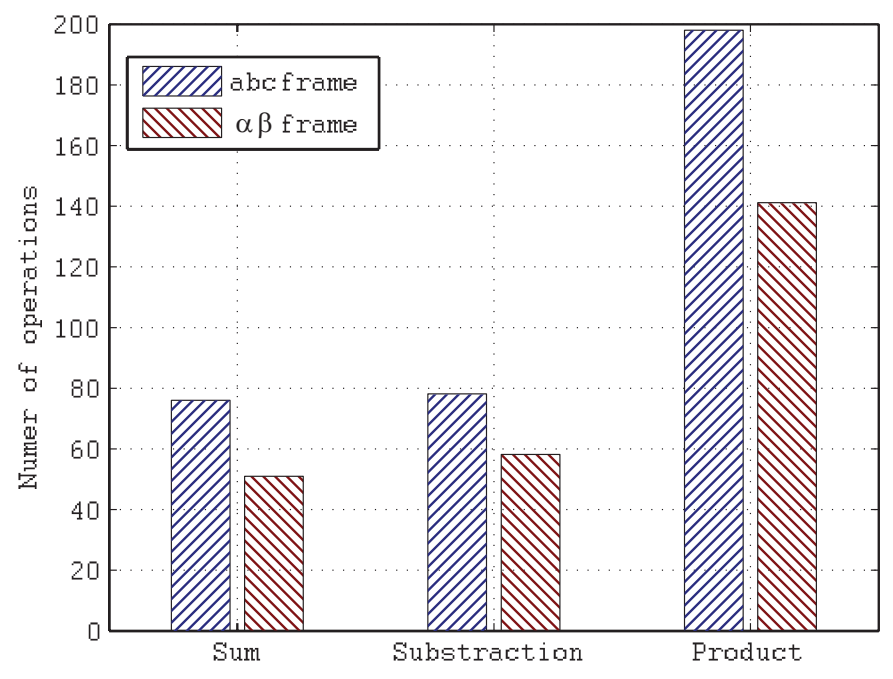

Fig. 8. Number of operation comparison.

the high order switching frequency components. However, this filter makes the controller design stage more complex. In this papers two algorithms to develop a FS-MPC control of the VSI are compared. One expressed in the abc frame and other in the $\alpha-\beta$ frame. Their performances are appraised in terms of the system output voltage quality and computational cost of the algorithm. It has been demonstrated through simulations that both algorithms provide the same output voltage quality under linear and non-linear operation conditions. However, when the computational cost of the algorithms are evaluated it has been addressed that performing the algorithm in the $\alpha-\beta$ frame reduces the computational effort required to the control hardware. Thus, it is possible to choose a cheaper control hardware platform to implement the VSI controller.

\section{ACKNOWLEDGMENT}

The authors gratefully acknowledge the financial support provided by the Spanish government within the researching project TEC2007-61879, the Andalusian Government within the researching project P07-TIC-02991, the Chilean Research Fund CONICYT-FONDECYT (Grant 1080443), the University of Seville and by Universidad Tecnica Federico Santa Maria.

\section{REFERENCES}

[1] O. Kukrer, "Deadbeat control of a three-phase inverter with an output LC filter," IEEE Transactions on Power Electronics, vol. 11, no. 1, pp. 16-23, January 1996.

[2] M. Kojima, K. Hirabayashi, Y. Kawabata, E. C. Ejiogu, and T. Kawabata, "Novel vector control system using deadbeat-controlled PWM inverter with output LC filter," IEEE Transactions on Industry Applications, vol. 40, no. 1, pp. 162-169, January/February 2004.

[3] P. Mattavelli, "An improved deadbeat control for UPS using disturbance observers," IEEE Transactions on Industrial Electronics, vol. 52, no. 1, pp. 206-212, February 2005.

[4] P.C. Loh, M.J. Newman, D.N. Zmood, and D.G. Holmes, "A comparative analisys of multiloop voltage regulation strategies for single and threephase UPS systems," IEEE Transactions on Power Electronics, vol. 18, no. 5, pp. 1176-1185, September 2003. 
[5] P.C. Loh and D.G. Holmes, "Analysis of multiloop strategies forLC/CL/LCL-filtered voltage-source and current-source inverters,' IEEE Transactions on Industry Applications, vol. 41, no. 2, pp. 664 654, March/April 2004.

[6] S. Buso, S. Fasolo, and P. Mattavelli, "Uninterruptible power supply multiloop control employing digital predictive voltage and current regulators," IEEE Transactions on Industry Applications, vol. 37, no. 6, pp. 1846-1854, November/December 2001.

[7] A. Kulka, T. Undeland, S. Vazquez, and L. G. Franquelo, "Stationary frame voltage harmonic controller for standalone power generation," in European Conference on Electronics and Applications(EPE'07), pp. 110, Aalborg, Denmark, 2-5 September 2007.

[8] M.N. Marwali and A. Keyhani, "Control of distributed generation systems-part i: Voltages and currents control," IEEE Transactions on Power Electronics, vol. 19, no. 6, pp. 1541-1550, November 2004.

[9] G. Escobar, A.A. Valdes, J. Leyva-Ramos, and P. Mattavelli, "Repetitivebased controller for a UPS inverter to compensate unbalance and harmonic distortion,” IEEE Transactions on Industrial Electronics, vol. 54, no. 1, pp. 504-510, February 2007.

[10] G. Escobar, P. Mattavelli, A. M. Stankovic, A. A. Valdez, and J. LeyvaRamos, "An adaptive control for UPS to compensate unbalance and harmonic distortion using a combined capacitor/load current sensing," IEEE Transactions on Industrial Electronics, vol. 54, no. 2, pp. 839-847, April 2007.

[11] G. Willmann, D.F. Coutinho, L.F.A. Pereira, and F.B. Libano, "Multipleloop H-infinity control design for uninterruptible power supplies," IEEE Transactions on Industrial Electronics, vol. 54, no. 3, pp. 1591-1602, June 2007.
[12] R. Kennel and A. Linder, "Model predictive control for electrical drives," IEEE Power Electronics Specialist Conference (PESC'05), pp. 17931799, Recife, Brazil, 12-16 June 2005.

[13] P. Cortes, M. P. Kazmierkowski, R. M. Kennel, D. E. Quevedo, and J. Rodriguez, "Predictive control in power electronics and drives," IEEE Transactions on Industrial Electronics, vol. 55, no. 12, pp. 4312-4324, December 2008

[14] S. Kouro, P. Cortes, R. Vargas, U. Ammann and J. Rodriguez, "Model predictive control-A simple and powerful method to control power converters," IEEE Transactions on Industrial Electronics, vol. 56, no. 6, pp. 1826-1838, June 2009

[15] M. Veenstra and A. Rufer, "Control of a hybrid asymmetric multilevel inverter for competitive medium-voltage industrial drives," IEEE Transactions on Industry Applications, vol. 41, no. 2, pp. 655-664, March/April 2005.

[16] P. Cortes, J. Rodriguez, "Three-phase inverter with output LC filter using predictive control for UPS applications," in European Conference on Electronics and Applications, Aalborg, Denmark, 2-5 September 2007.

[17] P. Cortes, G. Ortiz, J. Yuz, J. Rodriguez, S. Vazquez, and L. Franquelo, "Model predictive control of an inverter with output LC filter for ups applications," IEEE Transactions on Industrial Electronics, vol. 56, no. 6, pp. 1875-1883, June 2009

[18] K. J. Åström, "Computer-controlled Systems: theory and design," Prentice Hall, 3rd Ed., 1997.

[19] Reference guide: TMS320C28x Floating Point Unit and Instruction Set, www.ti.com, August 2008. 